\title{
Comparing Clinical Tests of Visual Loss in Cataract Patients Using a Quantification of Forward Light Scatter
}

\author{
D. B. ELLIOTT, M. A. HURST, J. WEATHERILL \\ Bradford
}

The decision to operate on patients with dense cataract and poor visual acuity (VA) is seldom difficult. A significant number of patients with cataract however retain relatively good VA yet have severe visual problems. ${ }^{1}$ Many authors have suggested that VA should be complemented by contrast sensitivity (CS) and glare disability (GD) testing in such patients. ${ }^{2-6}$ The American Academy of Ophthalmology have recently published a very useful review of the value of CS and GD testing in evaluating anterior segment disease, and in particular, cataract. ${ }^{7}$ The report suggests that $\mathrm{CS}$ measurements may be of value only as part of a battery of tests which must include some evaluation of neural integrity behind the cataract. It also indicates that a substantial amount of research is needed in the area of GD testing before it can be used as an established standard for indicating the need for cataract surgery. In particular, it recommends that the many commercially available GD tests would be best assessed by correlating their results with a quantification of overall visual performance. Probably the simplest way to evaluate overall visual performance is by using a questionnaire concerning how patients believe their vision affects their performance in everyday activities. Such questionnaires have been used with cataract, ${ }^{1,6}$ glaucoma ${ }^{9}$, and ARMD $^{10}$ studies. No standard technique is available, however, and also this type of evaluation is time consuming and requires considerable patient co-operation. An alternative assessment used by a

number of authors has been to compare GD test results with either VA or CS scores obtained outdoors. ${ }^{11-13}$ Similarly, many aspects of this type of evaluation need standardising, such as the type of test which should be used outdoors and the outdoor lighting levels and conditions. ${ }^{13}$

Visual loss in cataract is predominantly caused by light scatter. ${ }^{14}$ This produces a veiling luminance over the retina which reduces the contrast of the retinal image. It is because VA measurements do not always accurately reflect the effect of light scatter on visual function than CS and GD tests have been proposed as complementary tests in the evaluation of cataract patients. A simplier and more immediate strategy for comparing the value of different CS and GD tests in cataract evaluation, may therefore be to use a measure of light scatter as the 'gold standard' rather than some assessment of overall visual performance. Backward light scatter is the light scattered back from the ocular media which can be seen on slit-lamp examination. Objective measures of backscatter which are currently available, such as Scheimpflug slitimage photography and the Interzeag Opacity Lensmeter 701 , are only accurate at assessing the increased light scatter caused by ageing and by nuclear cataract. ${ }^{15,16}$ In any case, it is clearly more important to measure forward light scatter (that which is projected towards the retina), as it is this which produces the veiling luminance and subsequent visual loss. There is no conclusive evidence that there is 
an exact relationship between forward and backward light scatter. ${ }^{17,18}$

Presently, forward light scatter can only be measured in vivo using the direct compensation method developed by van den Berg. ${ }^{19-22}$ The technique has already been used to show the increase in light scatter with age, ${ }^{19}$ cataract, ${ }^{20,21}$ corneal dystrophy and hypopigmentation. ${ }^{21,22}$ In this study we measured light scatter using the direct compensation technique in 40 patients with cataract but no other ocular abnormality, and compared the results with clinical measurement of VA, CS and GD.

\section{Subjects}

Data were collected from 40 cataract patients (mean age 67.9 \pm 6.5 years) undergoing an anti-cataract drug trial at the University of Bradford Clinical Vision Research Unit. All subjects had been screened for ocular disease by direct and indirect ophthalmoscopy after dilation, slit-lamp biomicroscopy and applanation tonometry. Subjects with intraocular pressure greater than $21 \mathrm{mmHg}$ or with any ocular disease other than cataract were excluded. Subjects with poor general health, diabetes, a refractive error greater than $\pm 6.00 \mathrm{D}$, any history of amblyopia or ophthalmic surgery, and a logMAR VA of worse than 0.6 (Snellen equivalent 6/24) were excluded. To ensure that any loss of visual function was due purely to lens opacity, quantitative assessment of neural function behind the cataract was made using the Rodenstock retinometer ${ }^{2.3}$ and a hyperacuity technique. ${ }^{24.25}$ Displacement threshold hyperacuity measurements have been shown to be unaffected by the presence of mild cataracts, ${ }^{24}$ yet are sensitive to retinal and neural abnormality. ${ }^{25}$ Patients were excluded with a retinal VA of 0.63 (Snellen equivalent 6/9) or less, or a displacement threshold hyperacuity greater than 50 seconds of arc.

\section{Methods}

All measurements were made using natural pupils. LogMAR VA was measured at $4 \mathrm{~m}$ after a full refraction at a mean luminance of $160 \mathrm{~cd} / \mathrm{m}^{2}$ using the Ferris-Bailey chart. ${ }^{26}$ This chart has several advantages over the traditional Snellen chart. It has five letters on every line, with lines of equal legibility and a logarithmic progression in size from one line to the next.

Hess and $\mathrm{Woo}^{2}$ suggested that narrow angle scatter would affect CS only at the high spatial frequencies, and large angle scatter would reduce CS across.all frequencies. CS loss in cataract patients has been shown to be of these two distinct types. ${ }^{2.5}$ While the magnitude of the high frequency loss has been shown to be predictable from logMAR VA measurements, ${ }^{5} \mathrm{CS}$ loss at low frequencies is not. ${ }^{2.5}$ CS testing in cataract patients should therefore be made at low spatial frequencies. CS was measured using the Pelli-Robson letter CS chart $^{27}$ (see Fig. 1) at $1 \mathrm{~m}$ at the recommended luminance of $85 \mathrm{~cd} / \mathrm{m}^{2}$ Pelli et al. ${ }^{27}$ suggest that when used at $1 \mathrm{~m}$ the chart gives an indication of CS between 1-2 c/deg. The subjects read the letters, starting with those of high contrast until no letters in a given triplet could be identified. Credit was given $(0.05 \mathrm{log}$ units) for each individual letter read correctly, as this by-letter scoring rule has been shown to be more reliable than the original suggested by Pelli et al. ${ }^{28}$ To balance the legibility of different letters on the Pelli-Robson chart, any miscalling of the letter ' $\mathrm{C}$ ' as an ' $\mathrm{O}$ ' was counted as a correct call. ${ }^{29}$ All subjects were encouraged to look at a line for at least 20 seconds, as this is often needed before letters near contrast threshold can be perceived. Most measurements take about three minutes to complete.

Because contrast reduction by veiling glare has a relatively weak effect on VA measurements, GD is best measured as the difference in CS with and without a glare source. ${ }^{7}$ GD was measured as the reduction in Pelli-Robson CS when the patient's vision was hindered by the Brightness acuity tester (BAT) glare source. ${ }^{11}$ The BAT is a hand-held instrument which consists of a hemispherical bowl with an illuminated surface which contains a central $12 \mathrm{~mm}$ aperture. CS was remeasured with the patient looking through the BAT aperture with the bowl illuminated at the medium intensity setting of $300 \mathrm{~cd} / \mathrm{m}^{2}$. The high intensity setting has been reported to give innacurately high predictions of GD. ${ }^{12,13}$

The VA and CS tests used in this study conform to the guidelines of the American Aca- 
demy of Ophthalmology report, in that they both use the forced-choice procedure of letter identification, targets follow a uniform logarithmic progression, and the procedures are highly repeatable. ${ }^{28}$

Forward light scatter was measured using a portable version of the stray light meter developed by van den Berg. ${ }^{19-22}$ The patients position their eye against a cup at the top of a viewing tube. They view a circular target $1^{\circ}$ radius, surrounded by a circular ring with outer radius of $2^{\circ}$ of steady luminance of 30 $\mathrm{cd} / \mathrm{m}^{2}$. Concentric to this target and positioned along the inside of the viewing tube are three rings of yellow (lambdamax 570 $\mathrm{nm}$ ) light emitting diodes at angular distances from the patient's eye of $3.5,10$ and 28 degrees. The LED sources flicker sinusoidally at $8 \mathrm{~Hz}$. The three rings can be illuminated separately to allow measurement of light scatter at each of three angular distances. The subject is instructed to observe the central target, and one of the three rings is switched on. Due to forward light scatter within the eye, a visible flicker is seen on the central target. The investigator then slowly increases the luminance modulation of the central target which flickers in counterphase to the LED sources. The amplitude of this luminance modulation which produces zero perceived flicker corresponds directly to the amount of forward light scatter $^{19-22}$. As the central target is large and the task is to perceive a flickering stimulus, refractive blur has little effect on the measurements. ${ }^{19-22}$ For each of the three scattering angles, six measurements were made and an average taken. The investigator increases the luminance modulation of the central target and records the disappearance point of the central flicker percept and the reappearance point where the target modulation overwhelms the stray light scatter. This was

Table I Showing means ( \pm I $S D)$ in log units of $\log M A R V A$, Pelli-Robson $C S, G D$ and light scatter scores at angles of 3.5, 10 and 28 degrees of 40 cataract patients.

\begin{tabular}{rlll}
\hline LogMAR VA $:$ & $0.17 \pm 0.15$, & range & -0.14 to 0.40 \\
Pelli-Robson CS $:$ & $1.51 \pm 0.27$, & range 1.95 to 0.45 \\
GD $:$ & $0.20 \pm 0.18$, & range & -0.15 to 0.60 \\
3.5 degrees $:$ & $1.57 \pm 0.31$, & range 1.01 to 2.19 \\
10 degrees $:$ & $1.53 \pm 0.35$, range 0.86 to 2.24 \\
28 degrees $:$ & $1.39 \pm 0.32$, range 0.70 to 2.13 \\
\hline
\end{tabular}

repeated three times. ${ }^{19-22}$ The meter therefore uses a criterion-dependent psychophysical method of threshold determination.

The light scatter scores given subsequently are calculated from the equation $\mathrm{s}=\log [\mathrm{L}$ $(\Theta){ }^{*} \Theta^{2} / E(\Theta]$, where $L$ is the compensating luminance modulation of the central target, $\Theta$ is the scattering angle and $\mathrm{E}$ is the illuminance of the straylight source measured at the pupil plane. ${ }^{19-22,30}$

In a pilot study we calculated the standard deviation of the difference between test and retest data from 22 normal subjects for the $3.5,10$ and 28 degree scattering angles and obtained values of $\pm 0.07, \pm 0.08$ and \pm 0.14 log units respectively (Pearson correlation coefficients of test-retest data are $0.83,0.76$ and 0.69 respectively). The wide angle straylight source was positioned very close to the subject's eye at the end of the viewing tube. Slight changes in the position of the subject's eye could alter the straylight score at this angle and this may explain it's poorer repeatability.

\section{Results}

The mean and range of values in log units of logMAR VA, Pelli-Robson CS and GD and the three straylight scores are shown in Table I. Pearson correlation coefficients between forward light scatter at 3.5, 10 and 28 degrees and each clinical test of visual function are shown in Table II. There was no significant correlation between VA and either CS $(r=0.29)$ or $\mathrm{GD}(\mathrm{r}=0.22)$.

\section{Discussion}

The correlation of logMAR VA against straylight scores is highest at the $3.4^{\circ}$ angle of scatter and is progressively less for the two wider angles (see Table II). This fits the hypothesis that high spatial frequency CS (and therefore $\mathrm{VA}$ ) is predominantly affected by narrow

Table II Showing Pearson correlation coefficients between forward light scatter in log units at 3.5, 10 and 28 degrees and $\log M A R$ VA, Pelli-Robson CS and GD.

\begin{tabular}{rrrr}
\hline & \multicolumn{3}{c}{ Forward light scatter } \\
\hline & 3.5 & 10 & 8 \\
logMar VA & $0.54^{* * *}$ & $0.45^{* *}$ & $0.36^{*}$ \\
Pelli-Robson CS & $-0.76^{* * *}$ & $-0.75^{* * *}$ & $-0.60^{* * *}$ \\
GD & $0.51^{* * *}$ & $0.56^{* * *}$ & $0.45^{* *}$ \\
\hline
\end{tabular}


angle light scatter. ${ }^{2,31}$ Pelli-Robson CS scores are equally correlated with the 3.5 and 10 degree straylight scores, which may reflect its purported measurement of CS at low and intermediate spatial frequencies. The correlations with the $28^{\circ}$ angle straylight scores were lowest for all tests. This may be due to the greater variability in straylight scores at this angle. The slightly lower mean straylight score at the $28^{\circ}$ angle (see Table I) also suggests that early cataract does not scatter as much light at such wide angles.

CS is shown to be better correlated with light scatter at all angles than VA. This is not surprising as the reduction in contrast caused by glare has a relatively weak effect on VA measurements. ${ }^{7}$ In addition, some patients in the study with extensive cortical cataract retained excellent VA by presumably looking through a 'hole' in the cataract. These patients showed high light scatter scores and poor CS.

CS was also shown to be better correlated with the light scatter than GD. GD tests are intended to enhance the effect of light scatter on clinical tests of visual function. Early tests tended to score GD as the Snellen VA or CS level when hindered by a nearly bright light source. ${ }^{32,33}$ Pearson correlation coefficients between Pelli-Robson CS under BAT glare conditions and light scatter scores at 3.5, 10 and 28 degrees in the present study were $r=0.82,-0.84$ and -0.67 respectively. When compared with the results shown in Table II, these values indicate that the glare source did enhance the effects of light scatter on CS. It is only recently that the difference in either VA or CS results when hindered by a glare source have been used as a measure of GD. The aim of this scoring system is to ensure that results reflect only the amount of light scattered by the cataract and are independent of the integrity of the underlying neural system. Unfortunately, this scoring system also has disadvantages. Firstly, the score is inherently twice as variable, as two measurements are used in its calaculation. Secondly, when measured in this way, GD is not used to enhance the effect of light scatter, but rather as a measure of light scatter in itself. In this study, therefore, for GD to have been a more accurate assessment of the effect of light scat- ter than the original CS score, the glare source would be expected to more than double the reduction in CS from its baseline value. For example, if a theoretical patient's baseline (without cataract) Pelli-Robson CS was 1.80 $\log$ units, and this was reduced to $1.50 \mathrm{log}$ units by cataract, then the GD needs to be at least $0.30 \log$ units for equal accuracy. The range of values shown in Table I shows that this was not the case in this study. Using a brighter glare source would have increased the range of scores and possibly improved the correlation of GD with light scatter. In previous work, however, we have used the BAT with the illuminance set at its highest setting of $1,000 \mathrm{~cd} / \mathrm{m}^{2}$, and this washed out all the letters on the Pelli-Robson chart for a number of early cataract patients. Because the patient's original reduced CS (or VA) is used as a starting point, the possible range of scores is restricted. The BAT high intensity setting has also been reported to give inaccurately high predictions of GD when compared with GD assessment in bright sunlight outdoors. ${ }^{12,13}$

It would be interesting to compare straylight scores from cataract patients with an assessment of overall visual performance. Although this study did not attempt to do this, the coefficient values shown in Table II can be compared to results of monocular scores of the same tests correlated with quantified perceived visual disability reported previously. ${ }^{6}$ The pattern of results is similar.

\section{Conclusion}

Visual loss in cataract is predominantly caused by forward light scatter. It is because VA measurements do not always accurately reflect the effects of light scatter on visual function, that CS and GD testing has been advocated in assessing early cataract. We propose the measurement of forward light scatter as a 'gold standard' with which to compare clinical tests of visual function in cataract patients. In this study we have found a much higher correlation between light scatter and Pelli-Robson CS than between light scatter and either $\log$ MAR VA or GD measured using the Pelli-Robson chart and the BAT. CS and GD tests provide supporting psychophysical evidence for the need for surgery in cataract patients who retain relatively good 
VA. In such patients, it is generally possible to obtain an ophthalmoscopic view of the fundus and possibly an assessment of central visual fields. If such patients appear to have normal neural function then the present study indicates that Pelli-Robson CS measurements, particularly when hindered by the BAT glare source, provide an accurate assessment of the amount of forward light scatter within the eye. The one disadvantage of CS 'measurement to evaluate visual loss in cataract patients is that CS is dependent on the integrity of the neural as well as optical systems. The decision to operate on cataract patients with relatively good VA who also have some neural abnormality is more complex. A number of tests are available to evaluate retinal function behind cataract, ${ }^{23}$ and these should be used in such cases. Measurements of GD (calculated as the difference in VA or CS) may help, but because of the problems discussed above, GD scores should not be used alone, but as part of a battery of tests.

The portable straylight meter used in this study was a first prototype and at present is not suitable for clinical use. With the modifications which are planned, however, it may prove itself to be a very useful clinical instrument.

We would like to thank Dr TJTP van den Berg and Dr JK Ijspeert for the loan of their Straylight meter, and for helpful comments.

\section{References}

${ }^{1}$ Bernth-Petersen P: Visual functioning in cataract patients. Acata Ophthalmol 1981, 59: 198-205.

${ }^{2}$ Hess R and Woo G: Vision through cataracts. Invest Ophthalmol Vis Sci 1978, 17: 428-35.

${ }^{3}$ Paulsson LE and Sjöstrand $\mathrm{J}$ : Contrast sensitivity in the presence of a glare light. Invest Ophthalmol Vis Sci 1980, 19: 401-6.

${ }^{4}$ Koch DD: Glare and contrast sensitivity testing in cataract patients. J Cataract Refract Surg 1989, 15: 158-63.

${ }^{5}$ Elliott DB, Gilchrist J, Whitaker D: Contrast sensitivity and glare sensitivity with three types of cataract morphology: are these techniques necessary in a clinical evaluation of cataract? Ophthal Physiol Opt 1989, 9: 25-30.

${ }^{6}$ Elliott DB, Hurst MA, Weatherill J: Comparing clinical tests of visual function in cataract with the patient's perceived visual disability. Eye 1990, 4: 712-17.

${ }^{7}$ American Academy of Ophthalmology: Contrast sensitivity and glare testing in the evaluation of anterior segment disease. Ophthalmology 1990, 97: 1233-7.
${ }^{8}$ Guidex GM and Kind P: The QALI tool kit. Discussion paper No. 38, Centre for health economics. York University, 1988.

${ }^{9}$ Ross JE, Bron AJ and Clarke DD: Contrast sensitivity and visual disability in chronic simple glaucoma. Br J Ophthalmol 1984, 68: 821-7.

${ }^{10}$ Lennerstrand $\mathrm{G}$ and Ahlstrom $\mathrm{CO}$ : Contrast sensitivity in macular degeneration and the relation to subjective visual impairment. Acta Ophthalmol 1989, 67: 225-33.

${ }^{11}$ Holladay JT, Prager TC, Truillo TC, Ruiz RS: Brightness acuity test and outdoor visual acuity in cataract patients. J Cataract Refract Surg 1987, 13: 67-9.

${ }^{12}$ Neumann AC, McCarthy GR, Locke J, Cobb B: Glare disability devices for cataractous eyes: A consumer's guide. J Cataract Refract Surg 1988, 15: $212-16$.

${ }^{1.3}$ Prager TC, Urso RG, Holladay JT, Stewart RH: Glare testing in cataract patients: Instrument evaluation and identification of sources of methodological error. J Cataract Refract Surg 1989, 15: 149-57.

${ }^{14}$ Philipson B: Light scattering in lenses with experimental cataract. Acta Ophthalmol 1969, 47: 1089-101.

${ }^{15}$ Chen SY, Chylack LT, White O: Topcon SL-45 photography. A suitable technique for documenting nuclear but not cortical cataractous change in vivo. Invest Ophthalmol Vis Sci (Suppl) 1985, 25: 119.

${ }^{16}$ Elliott DB and Hurst MA: Assessing the effect of cataract: A clinical evaluation of the Opacity Lensmeter 701. Optom Vis Sci 1989, 66: 257-63.

${ }^{17}$ Feuk T and McQueen D: The angular dependence of light scattered from rabbit corneas. Invest $\mathrm{Oph}$ thalmol Vis Sci 1971, 10: 294-9.

${ }^{18}$ Allen MJ and Vos JJ: Ocular scattered light and visual performance as a function of age. $A m J$ Optom 1967, 54: 717-27.

${ }^{19}$ van den Berg TJTP: Importance of pathological intraocular light scatter for visual disability. Doc Ophthalmol 1986, 61: 327-33.

${ }^{20}$ van den Berg TJTP, DeWard PWT, Ijspeert JK, de Jong PT VM: Intraocular light scattering assessed quantitatively in age related cataract. Invest $\mathrm{Oph}$ thalmol Vis Sci (Suppl) 1989, 30: 499.

${ }^{21}$ Ijspeert JK, DeWard PWT, van den Berg TJTP and deJong PTVM: The intraocular straylight function in 129 healthy volunteers; dependence on angle, age and pigmentation. Vision Res 1990, 30: 699-707.

22 van den Berg TJTP, Ijspeert JK, DeWard AWT, Meire F: Functional quantification if diaphany. Doc Ophthalmol 1990, 75: 239-46.

${ }^{23}$ Fuller DG and Hutton WL: Presurgical Evaluation of Eyes with Opaque Media. London: Grune and Startton, 1982.

${ }^{24}$ Whitaker D and Elliott DB: Towards establishing a clinical displacement threshold technique to evaluate visual function behind cataract. Clin Vision Sci 1988, 4: 61-9.

${ }^{25}$ Whitaker D and Deady J: Prediction of visual function behind cataract using displacement threshold hyperacuity. Ophthal Physiol Opt 1989, 9: 20-5. 
${ }^{26}$ Ferris FL, Kassof A, Bresnick GH, Bailey I: New visual acuity charts for clinical research. Am J Ophthalmol 1982, 94: 91-6.

${ }^{27}$ Pelli DG, Robson JG, Wilkins AJ: The design of a new letter chart for measuring contrast sensitivity. Clin Vis Sci 1988, 2: 187-99.

${ }^{28}$ Elliott DB, Bullimore MA, Bailey IL: Improving the reliability of the Pelli-Robson chart. Optom Vis Sci (Suppl) 1990, 67: 176.

${ }^{29}$ Elliott DB, Whitaker D, Bonette L: Differences in the legibility of letters at contrast threshold using the Pelli-Robson chart. Ophthal Physiol Opt 1990, 10: 323-6.

31) Vos JJ: Disability glare: A state of the art report. CIE J 1984, 3: 39-53.

${ }^{31}$ Hemenger RP: Light scatter in cataractous eyes. Ophthal Physiol Opt 1990, 10: 394-6.

${ }^{32}$ Cinotti AA: Evaluations of indications for cataract surgery. Ophthal Surg 1979, 10: 25-31.

${ }^{33}$ Harbin TS: Visual impairment by sunlight in posterior subcapsular cataracts. Ophthalmic Surg 1973, 4: 34-6. 